Editorial

\title{
Awareness on pregnancy induced hypertension
}

\section{Editorial}

Pregnancy induced hypertension is a condition which brings bad effects many women in the world. It is an alarming condition which can affect both the welfare of the mother and the child. Women who develop high blood pressure in pregnancy (any trimester) appear to have an elevated risk high blood pressure and stoke in later life. Women with a history of high blood pressure in pregnancy, known as gestational hypertension, were more likely than women with no history to develop high blood pressure in later years. Literature suggests that pregnancy-induced hypertension (PIH) affects approximately one out of every 14 pregnant women. Although PIH more commonly occurs during first pregnancies, it can also occur in subsequent pregnancies. $\mathrm{PIH}$ is also more common in pregnant teens and in women over age 40. Many times, PIH develops during the second half of pregnancy, usually after the 20th week, but it can also develop at the time of delivery or right after delivery. To best of my knowledge recent status of gestational hypertension in our country is not known. This disease is responsible for high maternal and perinatal morbidity and mortality rates, and is one of the main public health problems. ${ }^{1,2}$ According to the Health Ministry, hypertension during pregnancy, depending on the severity level, is considered a risk factor, which, associated with individual characteristics, unfavorable socioeconomic conditions, certain obstetric histories and clinical problems could trigger harms to the maternal-fetal binomial. We know pregnant women and newborn baby are usually vulnerable and prone to suffer adverse condition. This is why awareness programme on pregnancy induced hypertension

\author{
Volume 5 Issue 2 - 2017
}

Monoarul Haque

Publication Secretary, Bangladesh Physiotherapy Association, Bangladesh

Correspondence: Monoarul Haque, Publication Secretary, Bangladesh Physiotherapy Association, Bangladesh, Email monoarmunna@yahoo.com

Received: January 29, 2017 | Published: February 08, 2017

is necessary so that we can prevent it and it is worth saying cure is never better than prevention. Counselling about risk factors, regular antenatal check-up can minimize this problem. Commencement of community based health education and health promotion is necessary.

\section{Acknowledgements}

None.

\section{Conflict of interest}

The author declares no conflict of interest. 